\title{
Flame retardants for polymer systems
}

\author{
Eli M. Pearce \\ Polymer Research Institute, Polytechnic University, \\ 333 Jay Street, Brooklyn, NY 11201, U.S.A.
}

\begin{abstract}
Mechanisms for flame retardation are reviewed and general principles developed. Approaches for utilizing flame retardants in various systems are discussed. Structure and flammability relationships are developed. Recent developments in flame retardants are presented. Market forecasts and directions are reviewed.
\end{abstract}

\section{INTRODUCTION}

Factors associated with polymer flammability have been previously reviewed by us (refs. 1 and 2). There are a number of stages that one can use to simply describe the polymer flammability process. These stages are a) preheating, b) decomposition to give volatile compounds and residuals, c) ignition of the volatiles in the presence of oxygen, and d) combustion. This process is a sustained exothermic cyclic process and mechanisms to decrease the flammability of a polymer involve interrupting the cyclic process at some stage. We have elucidated a number of basic mechanistic concepts and related chemistry which are important for the understanding to reduce flammability. We will discuss this aspect in regard to flame retardants available commercially and also in terms of future directions for new and improved flame retardants. At the same time one must also consider other aspects in the development of flame retardants certainly not the least of which is the economic viability, the processibility, and the effect on other properties.

An ideal flame retardant polymer system should have high resistance to ignition and flame propagation, a low rate of combustion, a low rate and amount of smoke generation, low combustibility and toxicity of combustion gases, no change in flammability during use, performance of the base material should be fairly close to the original and that there not be a difference in appearance, and that the economic penalty be acceptable.

To this date most flame retardant systems have incorporated either chlorine or bromine, phosphorus, antimony and boron related compounds. Combinations of these and synergistic effects, for example, the antimony oxide halogen synergism have been described in the literature. What will become apparent in this paper is that there are other approaches that can be considered which might not contain these particular elements. Practically all commercial plastics are "compounded" with various additives to improve their processing and in use performance. Other than plasticizers, flame retardants is probably the largest volume additive to be used in polymeric materials.

\section{TECHNIQUES FOR FLAME RETARDANT APPLICATION OF POLYMER MODIFCATION}

In considering flame retardants and their structure, one has to consider how that additive is placed into the polymer structure or final product and in addition one has to consider how this flame retardant modification affects a number of properties and processing parameters. First let us consider the possible approaches to the desired modification.

If we were to think in terms of a textile modification, one could think of flame retarding a polymer system by using a monomer as a flame retardant and incorporating this as a copolymer in the system. One then could go through 
the polymerization process. If one wanted to think in terms of an additive, one would incorporate such an additive into the spinning liquid or in the case of a plastic material one could incorporate it as an additive into the mix that goes into an extruder. In the case of a fiber, chemical after-treatment could occur by grafting and one could think in terms of using a flame retardant here. In addition physical or chemical after-treatments with additives on the textile could also be an appropriate approach. Depending on where the application of the flame retardant is made we could expect various properties and utility. For example, if the flame retardant is part of the polymer chain, advantages could possibly include excellent durability and aesthetics, and disadvantages could be cost, probably the development of some new technology, and possibily lower processing efficiency. When one thinks of a chemical retardant additive in the fiber, there could be cost advantages, perhaps more durability than a topical treatment, and the disadvantages might be related to the complexity of the technology, as well as the intermediate durability in use. If one used a topical treatment of a fabric the advantages are that the cost and technology are probably quite available and useful and the disadvantages that there is potential loss of retardancy in use and aesthetics. Also there may be certain finishing plant polution control problems that one has to contend with.

One also has to consider the properties that are changed in the systems. For example, incorporation of a comonomer into a polymer system that relies in some large degree on the crystallinity of that polymer for its properties, would have a negative effect on this because the crystallinity, would decrease as a function of increasing comonomer concentration. If one just considered the additive approach, one has to consider whether the additive will behave generally as a filler or as a plasticizer. If the flame retardant in the polymer system behaves as a plasticizer, we would expect reductions in melt viscosity, perhaps crystallinity and decreased tensile strength and modulus with some increase in elongation.

If the material that is used behaves as a filler we must consider how to obtain good bonding between the filler surface and the polymer so that mechanical properties can be maximized. Now these properties will usually show increased modulus and perhaps an increase in tenacity but usually a decrease in elongation and impact strength. Melt viscosity will usually increase. We seldom think in terms of these effects on properties in designing our flame retardants and I propose that this is an area where there is still great opportunity for designing flame retardants with specific effects on polymer properties other than the flame retardancy itself.

Needless to say, factors that must continuously be looked at are safety and health. We have had a number of situations in the flame retardance area where there has been cause for problem and I suspect this may occur again. There are certainly two general approaches that should be considered and that is that safety and health problems diminish if a polymer additive is reacted into the polymer system or if the polymer additive is of sufficiently high molecular weight that it does not volatize and/or readily diffuse out of the polymer system. In both of these cases, this will decrease safety and health problems and as we shall see a number of present day approaches are based on either polymeric additives, additives of relatively high molecular weight, or additives that are reacted into the polymer system itself. There also has been some unusually promising developments in some cases where additives inherently do not appear to be of any potential safety or health problem.

It is interesting that most companies manufacturing materials for flame retardants now provide thermogravimetric analysis data which is useful for the selection of the appropriate flame retardant for a particular polymer system (ref. 3). This information is useful in two ways. The first is that for developing a process in which this flame retardant is now used, knowing its degradation profile you will know whether it will be able to survive the temperature of any of your processing steps. Obviously if it is not able to do that it would be an inappropriate flame retardant for your system. The second is that generally flame retardants decrease the thermal stability of your system. It is important that by whatever the mechanisms, that these mechanisms occur before the polymer you are flame retarding degrades. Thus one tries to match thermogravimetric analysis curves so that the flame retardant degrades just a little before the polymer that is being flame retarded. The efficiency of matching the degradation curves is a key to effective flame retardancy. If the flame retardant additive possesses too 
low a thermal stability compared to that of the polymer, it will be lost before its function is needed. If the additive has greater stability, it may remain intact at the time its function is needed.

\section{SOME FLAMMABILITY-STRUCTURE RELATIONSHIPS (ref. 1)}

In utilizing flame retardants, there are some general relationships that we can understand. Many polymers can be degraded at relatively low temperatures, for example, at less than $400^{\circ}$ to give monomer, dimer, or other low molecular weight combustible volatiles and will have low oxygen index values. Thus polymers like polyethylene, polypropylene, polyformaldehyde, polystyrene, and polymethylmethacrylate have oxygen indices of less than 21. Polymers having aromaticity in the backbone usually have improved oxygen indices. For example, the aramid, Nomex, has an oxygen index of 32 while nylon 66 has an oxygen index of 23 . The presence of certain flame retarding elements such as halogen or phosphorus will also increase the oxygen index. Thus, polyvinylchloride has an oxygen index of 45. Polymers which contain little or no hydrogen or contain very strong bonds or degrade to monomer that is relatively non-flammable also have a high oxygen index, such as polytetrafluoroethylene $(\mathrm{OI}=95)$.

Our studies have shown that end groups in certain polymer systems such as nylon 6 and polyethyleneterephthalate can have some effect on the flammability of the polymers. The thermal degradation of these polymers can be end group catalized. So for example in the case of nylon 6 , increasing amounts of amine and carboxylic acid end groups decrease the oxygen index whereas in polyethyleneterephthalate there is a decrease as the carboxylic acid end groups increase.

We have also shown that when certain flame retardant structures are used as a comomomer versus being used as an additive, it is difficult to predict in advance whether there will be an improvement in flammability behavior. For example, tetrabrombisphenol-A incorporated as an additive into polyethyleneterephthalate versus its use as an equivalent comomoner showed little or no difference in oxygen index. In contrast when triphenylphosphine oxide was added to polyethyleneterephthalate it showed vapor phase activity and an increasing oxygen index. When the dicarboxylic acid analog of triphenylphosphine oxide was used and incorporated into polyethyleneterephthalate, it now also showed an increase in oxygen index but the phosphorus apparently behaved as a condensed phase flame retardant. Thus depending on the volatility of the moiety added, one can actually obtain a switch in mechanism.

We have concentrated our studies on the area of char formation. In order to increase char formation in a polymer system one has to promote the formation of a reasonable number of thermally stable crosslinks and/or the formation of very high temperature stable ring structures. These principles have been demonstrated by us in several ways. For example, polystyrene when modified with vinylbenzyl chloride in the presence of either antimony oxide or zinc oxide showed a high degree of char formation and crosslinking. We proposed the formation of Lewis acids which promoted Friedel-Crafts alkylation reactions. Another system studied by us which showed similar increased char formation was that of polymers containing cardo(looped functionality) units. For example, systems based on phenolphthalein such as epoxies, polycarbonates or polyesters, showed enhanced crosslinking and increased char formation. We demonstrated that the crosslinking reaction was due to the opening of the lactide group of the phenolphthalein to give ester groups crosslinking between chains. A large number of other cardo structures were also shown to give these enhanced char formation reactions.

Our studies on substituted aromatic polyamides were examples in which high temperature thermally stable aromatic rings were formed. We have investigated orthohalogen substituted aramids and have shown that at elevated temperatures benzoxazole structures were formed. The amount that was formed at these elevated temperatures was dependent on the nature of the halogen groups.

Additional studies by us on styrylpyridine-based polymers were examples where we demonstrated that both crosslinking and ring formation occurred. number of systems prepared by us containing the styrylpyridine structures also showed enhanced char formation. 


\title{
RECENT DEVELOPMENTS IN FLAME RETARDANTS
}

\author{
Halogen containing flame retardants \\ "Dechlorane plus" continues to be a very popular flame retardant usually \\ compounded with antimony oxide to maximize reduced flammability. Most \\ recently data has been published which indicates that additives such as zinc \\ borate in combination with antimony oxide can produce low smoke \\ formulations. In addition a finely ground free flowing power form has \\ become available and this apparently leads to improved properties for films, \\ coatings, and adhesives. Also formulations which show improved flame \\ retardancy and tracking have been developed for materials such as nylon and \\ show improvements over standard additives such as zinc oxide in glass \\ reinforced nylon samples. This particular combination is considered to be a \\ trade secret.
}

Of the halogen containing additives, bromine containing additives are still under serious development and modification. Recent reviews by R. C. Nametz (ref. 4,5) discuss the current status of the systems for thermoplastic and thermoset resins. These reviews consider the bromine containing flame retardants utilized in high impact polystyrene, polystyrene foam, ABS resins, polyolefins, nylon resins, poly (butylene terephthalate), unsaturated polyesters, epoxy resins, rigid polyurethane foams and flexible polyurethane foams.

Nametz has summarized the trends in flame retardant technology. It is predicted that there will be advanced second generation products with increased permanence. Halogen compounds will continue to play a very important role and monomers will be incorporated into the thermoplastic structures. There will be a concentration on having flame retardants which also bring other needed and desired properties to the polymeric system. This will then effect the economics in an appropriate manner. There will be increased emphasis on smoke and toxicity and there will be continued research to understand the combustion mechanisms and ways of inhibiting combustion. Our work (ref.1) has indicated that routes to this should emphasize char formation and mechanistically this would mean systems capable of forming high temperature stable crosslinks and systems capable of forming high temperature stable ring structures.

A recent study (ref. 6) has been published comparing poly (pentabromobenzyl acrylate) as a flame retardant for engineering thermoplastics. This flame retardant was compared to a number of bromine and chlorine containing systems in unreinforced and glass reinforced nylon 6 and PBT. It is claimed that this flame retardant improves compatiblity with fiber reinforcing agents, is a processing aid, shows improved weather resistance and is able to maintain good chemical resistance and electrical properties. The method of processing in a reinforced polymer is critical for the maintenance of mechanical properties and care must be taken that deterioration does not occur. Since this is a high molecular weight polymer, a real advantage for this system would be non-blooming.

Recently a brominated epoxy resin flame retardant for nylon has been reported (ref. 7). This is used in combination with antimony oxide and oxygen indices as high as 31 are reported.

\section{Phosphorus containing flame retardants}

Uses of phosphorus containing flame retardants have been reviewed recently (ref. 8). The classic arylphosphates and alkylphosphates in combinations are widely used in flame retarded flexible formulations and antimony oxide can be used as a synergist in PVC to also make improvements in flammability. Modified polyphenylene oxides are reported to be flame retarded with a phosphate ester. A reactive organic phosphorus compound containing hydroxy groups is reported to be a new approach to flame retarding epoxy resins. The structure of this compound is unavailable. Halogen and phosphorus containing compounds are used commercially in flexible and rigid polyurethanes. For example in a flexible urethane foam a blend of pentabromdiphenyl oxide and a phosphate ester is used. Phosphate ester, in addition to the reduced flammability, decreases the viscosity and the aromatic bromide compound has the advantage of producing a foam that does not scorch. Camino has recently studied intumescence in systems containing a polyfunctional alcohol, a catalyst such as ammonium polyphosphate, and a blowing agent. Formulations of this sort, seem to be effective in reducing the flammability of polymers such as polypropylene. 
Ethyl Corporation continues its development of aryloxyphosphazene materials that are halogen free, such as (phenoxy-p-ethylphenoxy) substituted phosphazenes, and reported to give an oxygen index of 28 (ref. 9).

\section{Other flame retardant systems}

Alumina trihydrate still is the most used flame retardant additive. It must be used in high loadings to achieve this flame retardancy.

Mechanisms for flame retardancy have primarily revolved around the loss of water, which is an endothermic process. The generated water is converted to steam and the steam also transfers heat away from the degradation sites. Because of the high loading it is essential that good bonding be obtained at the interface so that there can be retention of mechanical properties. Recent Union Carbide technology has shown that an improved alumina trihydrate using a coupling agent can make possible tough halogen free polyolefin composites. During degradation therefore corrosive acids will not be formed and the claim is made that there is significant reduction in smoke generation.

Most recently General Electric has been issued patents on SSR100 (ref. 10) silicon based flame retardant for polypropylene and other thermoplastics. This is an organosiloxane and may be used in combination with a halogen containing retardant or alumina trihydrate. AV-0 polypropylene has been obtained by use of such a flame retardant. Processing benefits are claimed for the system as well as nonblooming.

\section{MARKET CONSUMPTION OF FLAME RETARDANTS}

An assessment has been made comparing US consumption of flame retardants for 1984 and 1985 (ref. 11). It shows a modest increase from 453 million pounds to 460 million pounds. An increase in sales for bromine additives and a projected decrease for chlorinated additives is predicted. Non-reactive additives claimed about 399 million pounds whereas reactive additives claimed about 61.5 million pounds.

This reflects, in part, the lack of continued pressure on improving the flammability of polymeric materials in the United States. This normally would come from government agencies. The general political tenor at the moment is for less government involvement in setting such standards. This is a market very dependent upon the promulgation of standards. It is a fact that we possess the capabilities of making major improvements in the flammability reduction of many kinds of polymeric materials. The reason that this will not be done at any rapid pace is that there is no standard setting pressure and therefore little economic pressure. I believe that a way to move this field and have it advance is to adopt the same guidelines that were used for automobile mileage standards in the United States. That is to set up a number of standards to be met 5 years and 20 years from now and have industry now start the research to achieve these goals. If this were to be done, I believe we could make major advances in this area.

\section{REFERENCES}

1. E. M. Pearce, "Some Recent Aspects of Polymer Flammability," in Contemporary Topics in Polymer Science, Vol. V, Edited by E. J. Vandenburg, Plenum, New York, 1984. pps. 401-413.

2. Y. Khanna and E. M. Pearce, "Flammability of Polymers," in Applied Polymer Science, A.C.S. Symposium Series 285, R. W. Tess and G. W. Poehlein, eds., Am. Chem. Soc., Washington, D.C.. 1985. pps. 305-322.

3. E. M. Pearce, Y. P. Khanna, and D. Raucher, "Thermal Analysis in Polymer Flammability" in Thermal Characterization of Polymeric Materials, edited by E. Turi, Academic Press, New York, 1981, pps. 793-843.

4. R. C. Nametz, Plastics Compounding, 26-39, July-Aug. (1984) .

5. R. C. Nametz, Plastics Compounding, 54-66, Sept.-Oct. (1984).

6. S. Yanai, et. al., Plastics Compounding, 39-44, Nov.-Dec. (1985). 
7. I. Touval, Proceedings of the SPE 43rd Annual Technical Conference, 968-971, (1985).

8. J. Green, Plastics Compounding, 30-40, Nov.-Dec. (1984).

9. Reported in Modern Plastics, 12, May (1985).

10. R. R. Frye, U.S. Pat 4,387,176, (June 7, 1983) (to General Electric).

11. Reported in Modern Plastics, 65, Sept. (1985). 